\title{
Gastrointestinal Hemorrhage in Warfarin Anticoagulated Patients: Incidence, Risk Factor, Management, and Outcome
}

\author{
Wen-Chi Chen, ${ }^{1,2,3}$ Yan-Hua Chen, ${ }^{1}$ Ping-I Hsu, ${ }^{1,2}$ Feng-Woei Tsay, ${ }^{1,4}$ Hoi-Hung Chan, ${ }^{1,2}$ \\ Jin-Shiung Cheng, ${ }^{1}$ and Kwok-Hung Lai ${ }^{1,2}$ \\ ${ }^{1}$ Division of Gastroenterology, Department of Medicine, Kaohsiung Veterans General Hospital, 386 Ta-Chung 1st Road, \\ Kaohsiung 81362, Taiwan \\ ${ }^{2}$ Faculty of Medicine, School of Medicine, National Yang-Ming University, Taipei 11221, Taiwan \\ ${ }^{3}$ Department of Chemistry, College of Science, National Kaohsiung Normal University, Kaohsiung 82444, Taiwan \\ ${ }^{4}$ Department of Sport, Health \& Leisure, Cheng Shiu University, Kaohsiung 83347, Taiwan
}

Correspondence should be addressed to Yan-Hua Chen; fygmc@hotmail.com

Received 14 April 2014; Accepted 19 May 2014; Published 29 May 2014

Academic Editor: Seng-Kee Chuah

Copyright (C) 2014 Wen-Chi Chen et al. This is an open access article distributed under the Creative Commons Attribution License, which permits unrestricted use, distribution, and reproduction in any medium, provided the original work is properly cited.

Background. Warfarin reduces the incidence of thromboembolism but increases the risk of gastrointestinal bleeding (GIB). GIB during warfarin anticoagulation is rarely evaluated in Asian patients. Aims. This study aimed at investigating the incidence, risk factors, management, and outcome of GIB in Taiwanese patients treated with warfarin. Methods. We analyzed a cohort of warfarin anticoagulated patients between July 1993 and May 2012. Clinical data were retrieved in a chart-reviewing manner. Results. A total of 401 warfarin anticoagulated patients were enrolled. The incidence of GIB was 3.9\% per patient-years. Multivariate analysis with Cox regression showed that age $>65$ years old (RR: $2.5,95 \%$ CI: $1.2-5.5$ ), a mean international normalized ratio $>2.1$ (RR: $2.1,95 \%$ CI: 1.0 4.2), a history of GIB (RR: 5.1, 95\% CI: 1.9-13.5), and cirrhosis (RR: 6.9, 95\% CI: 2.0-24.5) were independent factors predicting GIB. $27.3 \%$ of the GIB patients had rebleeding after restarting warfarin while thromboembolic events were found in $16.7 \%$ of the patients discontinuing warfarin therapy. Conclusions. Warfarin was associated with a significant incidence of GIB in Taiwanese patients. The intensity of anticoagulation should be monitored closely during warfarin therapy, especially in patients with risk factors of GIB.

\section{Introduction}

Warfarin is currently the most commonly used oral anticoagulant worldwide. It produces an anticoagulant effect by interfering with the cyclic interconversion of vitamin $\mathrm{K}$ and its 2,3-epoxide (vitamin $\mathrm{K}$ epoxide) [1]. The indications of warfarin include prevention of venous thromboembolism, prevention of systemic thromboembolism and stroke in patients with prosthetic heart valves and atrial fibrillation, primary prevention of myocardial infarction, and prevention of stroke, recurrent infarction, and death in the management of acute myocardial infarction [2]. However, warfarin has a narrow therapeutic window, wide variability in doseresponse across individuals, and a significant number of drug and dietary interactions and requires close laboratory monitoring with frequent dose adjustment $[1,2]$.
Gastrointestinal bleeding (GIB) is one of the severe bleeding complications of warfarin anticoagulation and occurs in up to $12 \%$ of cases [3]. Several factors that influence the source and severity of GIB in patients taking warfarin are identified including prolonged prothrombin time, concomitant use of aspirin, advanced age, previous GIB, atrial fibrillation, and coexisting conditions such as renal insufficiency and anemia [4]. However, the correlation between some of these factors, for instance, advanced age, and GIB is controversial [5].

New oral anticoagulant agents are direct and selective inhibitors of a specific step or enzyme of the coagulation cascade. They have been shown to be effective in the prevention and treatment of various thromboembolic diseases with more predictable anticoagulant response and no need for close laboratory monitoring. However, new oral anticoagulants still have some limitations. Drug-drug 
interactions, difficulty in monitoring the anticoagulant effect in patients with severe renal and liver failure, the much more expensive prices compared with warfarin, and, most importantly, lack of a specific antidote are the major drawbacks of these agents [6]. A recent meta-analysis reveals that new generation of oral anticoagulants results in a significantly higher risk of GIB compared with warfarin [7]. Besides, new oral anticoagulants are not cost-effective when compared with warfarin in patients with atrial fibrillation [8]. Taking these together, warfarin remains a widely used anticoagulant before more promising agents are available. As a result, a more detailed understanding of the use of warfarin and its bleeding complication is necessary while managing the patients.

Average warfarin dose required to maintain the international normalized ratio (INR) between 2.0 and 3.0 is affected by ethnicity [9]. The maintenance doses of warfarin for the Japanese and the Chinese are about 30\% and 40\% lower than those of Caucasians, respectively [10]. Actually, genetic determinants of warfarin dosing may affect the effect of warfarin $[11,12]$. Several studies evaluated GIB complications associated with warfarin in Western countries but the data of Asian population was rarely reported. This study investigated the incidence, characteristics, risk factors, management, and outcome of GIB in Taiwanese patients treated with warfarin anticoagulation therapy.

\section{Materials and Methods}

2.1. Study Design. This was a retrospective study of a cohort of warfarin anticoagulated patients in Kaohsiung Veterans General Hospital. We searched the electronic medical records of all patients with prescriptions of warfarin between July 1993 and May 2012. Patients were enrolled if they met the following criteria: (1) age equal to or more than 20 years old and (2) taking warfarin for more than 6 weeks. We reviewed the medical records and retrieved the information including age, gender, the indications and duration of warfarin therapy, concomitant medication during anticoagulation such as antiplatelet agents, nonsteroid anti-inflammatory drugs, and steroids, comorbidity, and INR values during anticoagulation. We further divided all patients into GIB group and non-GIB group. For patients with GIB, the symptoms and signs of GIB at presentation, needs of transfusion, endoscopic findings and therapies, duration of discontinuation of warfarin, and outcome of the patients were recorded. This study was approved by the Institutional Review Board of Kaohsiung Veterans General Hospital (VGHKS12-CT11-01).

2.2. Definition. The average INR value was determined as the mean of all INR values measured during anticoagulation and before GIB, if present. GIB was defined as (1) clinically evident hematemesis, melena, and hematochezia, or positive for occult blood test, and (2) needs of transfusion of two or more units of packed red blood cells, or a decline in hemoglobin level of $2 \mathrm{~g} / \mathrm{dL}$ or greater, or a systolic blood pressure $<100 \mathrm{mmHg}$ in patients negative for evident signs of GIB or occult blood test.
2.3. Statistical Analysis. The primary aim of this study was the incidence of GIB in patients treated with warfarin. The secondary aims were the risk factors of GIB and the outcome of the patients with GIB. The incidence of GIB was calculated as values per patient-year in this cohort. Demographic data were compared between patients of GIB group and nonGIB group. Categorical data were compared using chisquare or Fisher's exact tests as appropriate. Continuous variables with normal distributions were compared using independent Student's $t$-test. Continuous variables without normal distributions were compared using Mann-Whitney $U$ test. A receiver operating characteristic (ROC) curve was used to determine the cut-off value of the mean INR which best discriminated GIB patients from non-GIB patients. Risk factors of GIB were examined by univariate and multivariate Cox regression analysis. Significance was defined as $P<0.05$ for all two-tailed tests. All analyses were conducted by using SPSS software (version 12; SPSS Inc., Chicago, IL).

\section{Results}

A total of 401 warfarin anticoagulated patients were enrolled into this study. There were 234 males and 167 females. The mean age of the patients was $65.2 \pm 16.6$ years. The indications of warfarin were as follows: valvular replacement in 148 patients (36.9\%), atrial fibrillation in 89 patients (22.1\%), deep vein thrombosis in 71 patients $(17.7 \%)$, pulmonary embolism in 31 patients $(7.7 \%)$, and other conditions in 62 patients (15.4\%). There were 36 patients in GIB group and 365 patients in non-GIB group. Demographic data of both groups were shown in Table 1 . Patients with GIB were older $(P<0.001)$ and had a higher mean INR value $(P=0.01)$ than patients without GIB. Besides, the proportion of a history of GIB $(P=0.003)$, concomitant cirrhosis $(P<0.001)$, and septic shock $(P<0.001)$ was also significantly higher in GIB group patients than in non-GIB group patients.

3.1. Incidence and Characteristics of GIB. Thirty-six patients had at least one episode of GIB. The incidence of GIB was $3.9 \%$ per patient-year. There were totally 43 bleeding episodes in this cohort and the average episode of GIB was 1.1 per patient. The time between the use of warfarin and the first onset of GIB was $41.0 \pm 58.4$ months. Twelve patients $(36.0 \%)$ had the first episode of bleeding within the first month after the start of warfarin anticoagulation. The presenting symptoms and signs of GIB included melena (15 patients, $41.7 \%$ ), hematemesis ( 9 patients, $25.0 \%$ ), hematemesis with melena (6 patients, $16.7 \%$ ), hematochezia (3 patients, $8.3 \%$ ), and a decrease of hemoglobin level more than $2 \mathrm{~g} / \mathrm{dL}$ but negative for evident signs of GIB or occult blood test (3 patients, $8.3 \%$ ).

Twenty-five of the 36 GIB patients underwent esophagogastroduodenoscopy (EGD) or colonoscopy according to the presentation of GIB. The causes of GIB included esophageal ulcer (1 patient, 4.0\%), gastric ulcer (1 patient, $4.0 \%$ ), duodenal ulcer (16 patients, $64.0 \%$ ), gastric polyp ( 1 patient, $4.0 \%$ ), rectal cancer ( 2 patients, $8.0 \%$ ), and pseudomembranous colitis (1 patient, 4.0\%) (Table 2). However, 
TABLE 1: Demographic data of patients treated with warfarin.

\begin{tabular}{|c|c|c|c|}
\hline Variables & Patients without $\mathrm{GIB}^{*} \quad(n=365)$ & Patients with $\mathrm{GIB}^{*} \quad(n=36)$ & $P$ value \\
\hline Age (year) & $64.1 \pm 17.0$ & $74.3 \pm 12.3$ & $<0.001$ \\
\hline Male & 215 & 19 & 0.5 \\
\hline Smoking & $101(27.6 \%)$ & $10(27.7)$ & 1.0 \\
\hline Alcohol & $73(20.0 \%)$ & $6(16.6 \%)$ & 0.6 \\
\hline \multicolumn{4}{|l|}{ Indications for warfarin } \\
\hline Deep vein thrombosis & $63(17.2 \%)$ & $8(22.2 \%)$ & 0.5 \\
\hline Pulmonary embolism & $28(7.6 \%)$ & $3(8.3 \%)$ & 0.9 \\
\hline Valvular replacement & $138(37.8 \%)$ & $10(27.7 \%)$ & 0.2 \\
\hline Atrial fibrillation & $81(22.1 \%)$ & $8(22.2 \%)$ & 1.00 \\
\hline Others & $55(15.0 \%)$ & $7(19.4 \%)$ & 0.5 \\
\hline Platelet count (K/cumm) & $201.6 \pm 139.2$ & $206.8 \pm 144.5$ & 0.9 \\
\hline Mean INR* value & $1.8 \pm 1.2$ & $2.2 \pm 1.7$ & 0.01 \\
\hline History of $\mathrm{GIB}^{\dagger}$ & $12(3.2 \%)$ & $5(13.8 \%)$ & 0.003 \\
\hline \multicolumn{4}{|l|}{ Comorbidity } \\
\hline Hypertension & $200(54.8 \%)$ & $22(61.1 \%)$ & 0.5 \\
\hline Cardiovascular disease & $62(16.9 \%)$ & $6(16.6 \%)$ & 1.0 \\
\hline Pulmonary disease & $32(8.7 \%)$ & $6(16.6 \%)$ & 0.1 \\
\hline Cirrhosis & $1(0.2 \%)$ & $3(8.3 \%)$ & $<0.001$ \\
\hline Renal insufficiency & $27(7.3 \%)$ & $4(11.1 \%)$ & 0.4 \\
\hline Malignancy & $38(10.4 \%)$ & $5(13.9 \%)$ & 0.6 \\
\hline Septic shock & $2(0.5 \%)$ & $3(8.3 \%)$ & $<0.001$ \\
\hline \multicolumn{4}{|l|}{ Concomitant medication } \\
\hline NSAID $^{\ddagger}$ & $6(1.6 \%)$ & $2(5.5 \%)$ & 0.1 \\
\hline Aspirin & $46(12.6 \%)$ & $4(11.1 \%)$ & 0.8 \\
\hline Clopidogrel & $30(8.2 \%)$ & $1(2.7 \%)$ & 0.2 \\
\hline Dipyridamole & $22(6.0 \%)$ & $5(13.8 \%)$ & 0.07 \\
\hline Steroids & $25(6.8 \%)$ & $4(11.1 \%)$ & 0.3 \\
\hline
\end{tabular}

* INR: international normalization ratio.

${ }^{\dagger}$ GIB: gastrointestinal bleeding.

${ }^{\ddagger}$ NSAID: nonsteroidal anti-inflammatory drug.

TABLE 2: Endoscopic findings of warfarin anticoagulated patients with gastrointestinal bleeding.

\begin{tabular}{lc}
\hline Source of bleeding & Patients (\%) \\
\hline Upper gastrointestinal bleeding & \\
Esophageal ulcer & $1(4.0 \%)$ \\
Gastric ulcer & $1(4.0 \%)$ \\
Duodenal ulcer & $16(64.0 \%)$ \\
Gastric polyp & $1(4.0 \%)$ \\
Lower gastrointestinal bleeding & \\
Rectal cancer & $2(8.0 \%)$ \\
Pseudomembranous colitis & $1(4.0 \%)$ \\
No identifiable source & $3(12.0 \%)$ \\
\hline
\end{tabular}

no identifiable source of bleeding could be found in 3 patients (12.0\%). One of these patients received both EGD and colonoscopy and the other two patients underwent EGD only because they refused colonoscopy. No enteroscopy or angiography was performed in these patients.

3.2. Risk Factors of GIB. We further stratified the patients according to the mean INR values and found that the incidence of GIB increased with higher intensity of anticoagulation (Table 3). A ROC curve found that a mean INR of 2.1 was the cut-off value which best discriminated patients with GIB from patients without GIB. Patients with advanced age also had a trend towards a higher incidence of GIB, especially in patients aged more than 70 years old (Table 4 ).

Univariate analysis with Cox regression showed that GIB was significantly related to age $>65$ years old (relative risk (RR): $2.5,95 \%$ confidence interval (CI): 1.3-5.3, $P=0.02$ ), a mean INR value $>2.1$ (RR: 2.4 , 95\% CI: $1.2-4.5, P=0.01$ ), a history of GIB (RR: 4.6, 95\% CI: $1.8-11.9, P=0.002$ ), and cirrhosis (RR: 8.9, 95\% CI: 2.7-29.9, $P<0.001$ ) (Table 5). Multivariate analysis with Cox regression revealed that age $>65$ years old (RR: $2.5,95 \% \mathrm{CI}: 1.2-5.5 ; P=0.02$ ), a mean 
TABLE 3: Incidence of gastrointestinal bleeding in relation to international normalization ratio.

\begin{tabular}{lcccc}
\hline INR $^{*}$ & Events & Patients & Follow-up (months) & Events/patient-years \\
\hline$\leq 1.0$ & 0 & 0 & 112 & 0 \\
$1.0-1.5$ & 6 & 5 & 3278 & 2.1 \\
$1.5-2.0$ & 18 & 14 & 5412.5 & 3.9 \\
$2.1-2.5$ & 11 & 9 & 2874 & 4.5 \\
$2.5-3.0$ & 2 & 2 & 887 & 4.5 \\
$>3.0$ & 6 & 6 & 503.1 & 14.3 \\
\hline
\end{tabular}

*INR: international normalization ratio.

TABLE 4: Incidence of gastrointestinal bleeding in relation to age.

\begin{tabular}{lcccc}
\hline Age & Events & Patients & Follow-up (months) & Events/patient-years \\
\hline$\leq 40$ & 0 & 0 & 869 & 0 \\
$41-50$ & 2 & 2 & 1273 & 1.8 \\
$51-60$ & 6 & 5 & 2336 & 3.0 \\
$61-70$ & 5 & 4 & 2115.5 & 2.8 \\
$71-80$ & 13 & 10 & 3667 & 4.2 \\
$>80$ & 17 & 15 & 2806.1 & 7.2 \\
\hline
\end{tabular}

INR > 2.1 (RR: 2.1, 95\% CI: 1.0-4.2; $P=0.04$ ), a history of GIB (RR: 5.1, 95\% CI: 1.9-13.5, $P=0.001$ ), and cirrhosis (RR: 6.9, 95\% CI: $2.0-24.5, P=0.003$ ) were independent factors predicting GIB after adjustment (Table 6).

3.3. Management of GIB and Outcome. Intravenous vitamin $\mathrm{K}$ was administered in all GIB patients. Patients were transfused with $2.8 \pm 5.0$ units of packed red cells and $2.7 \pm 6.3$ units of fresh frozen plasma. Eight patients underwent endoscopic treatments including hemoclipping (4 patients), endoscopic injection therapy (3 patients), and argon plasma coagulation (1 patient). Uncontrolled bleeding was noticed in 2 patients with a history of GIB and cirrhosis. Warfarin was restarted in 22 patients $(61.1 \%)$ in $7.9 \pm 6.5$ days after the GIB was controlled and none of these patients had thromboembolic events. However, recurrent GIB was found in 6 patients (27.3\%). Of these patients, 1 patient presented with recurrent gastric ulcer bleeding, 2 patients presented with recurrent duodenal ulcer bleeding, and 2 patients presented with gastric cancer bleeding. One patient was among the patients with a decrease of hemoglobin level of more than $2 \mathrm{~g} / \mathrm{dL}$ but negative for evident signs of GIB or occult blood test at the first bleeding episode and no bleeder could be identified at the rebleeding episode either. Warfarin was not restarted in 12 patients and none of them had recurrent GIB while cerebrovascular accident was noticed in 2 patients (16.7\%). Fourteen GIB patients died. The causes of mortality included sepsis (6 patients), pneumonia (2 patients), subdural hemorrhage (1 patient), heart failure ( 2 patients), acute myocardial infarction (1 patient), and uncontrolled GIB ( 2 patients). In all 401 patients, the incidence of cerebral vascular accident and myocardial infarction was $1.2 \%$ (5 patients) and $0.7 \%$ (3 patients), respectively.

\section{Discussion}

We investigated the incidence, risk factors, management, and outcome of GIB in a cohort of warfarin anticoagulated Taiwanese patients. GIB occurred at an incidence of 3.9\% per patient-year in this study. Age $>65$ years old, a mean INR value $>2.1$, a history of GIB, and cirrhosis were found to be independent risk factors of GIB. Warfarin was restarted in $61.1 \%$ of the GIB patients and $27.3 \%$ of them had recurrent GIB. Thromboembolic events were found in $16.7 \%$ of the patients who discontinued treatment of warfarin because of GIB.

GIB occurs at a rate ranging from $0 \%$ to $67 \%$ with an average bleeding rate of $3 \%$ in patients treated with warfarin [5]. The incidence of life-threatening and fatal hemorrhage episodes is around 5\% and 1\%, respectively [5]. Among our GIB patients, up to $33.3 \%$ of them had the first bleeding episodes within the first month of anticoagulation and $61.1 \%$ of the GIB occurred within the first year, which was consistent with the results of a meta-analysis [5]. This might be due to unstable intensity of anticoagulation during the early dosage adjustment period. As the INR values reach a stable therapeutic range, the bleeding incidence might decrease thereafter.

Polymorphisms in VKORC1 and CYP2C9 genes are associated with reduced doses of warfarin $[12,13]$. The effect of warfarin is affected by genetic determinants of warfarin dosing, which may vary between different races. In our study, the bleeding incidence was similar to those of Western studies. It is possible that the genetic polymorphisms are similar between different races so the dosing of warfarin may only play a partial role in addition to anticoagulation intensity, drug interactions, and underlying diseases. Recently, two large randomized controlled trials compared clinically 
TABLE 5: Univariate analysis of risk factors of gastrointestinal bleeding in patients taking warfarin.

\begin{tabular}{|c|c|c|c|}
\hline Variable & Relative risk & 95\% confidence interval & $P$ value \\
\hline Age $>65$ yrs & 2.5 & $1.3-5.3$ & 0.02 \\
\hline Mean INR ${ }^{*}>2.1$ & 2.4 & $1.2-4.5$ & 0.01 \\
\hline History of $\mathrm{GIB}^{\dagger}$ & 4.6 & $1.8-11.9$ & 0.002 \\
\hline Cirrhosis & 8.9 & $2.7-29.9$ & $<0.001$ \\
\hline Gender & 0.6 & $0.3-1.2$ & 0.2 \\
\hline Smoking & 1.0 & $0.5-2.3$ & 0.8 \\
\hline Alcohol & 0.7 & $0.3-61.8$ & 0.5 \\
\hline Hypertension & 0.6 & $0.8-3.3$ & 0.1 \\
\hline Cardiovascular disease & 1.5 & $0.7-3.1$ & 0.2 \\
\hline Pulmonary disease & 1.3 & $0.5-3.2$ & 0.5 \\
\hline Renal insufficiency & 1.1 & $0.4-3.2$ & 0.8 \\
\hline Malignancy & 1.2 & $0.5-3.0$ & 0.3 \\
\hline Septic shock & 3.0 & $0.9-10.1$ & 0.08 \\
\hline NSAID $^{\ddagger}$ & 3.0 & $0.7-12.7$ & 0.1 \\
\hline Aspirin & 0.9 & $0.3-2.7$ & 1.0 \\
\hline Clopidogrel & 0.5 & $0.07-3.9$ & 0.5 \\
\hline Dipyridamole & 2.4 & $0.9-6.2$ & 0.08 \\
\hline Steroids & 1.6 & $0.5-4.6$ & 0.4 \\
\hline
\end{tabular}

${ }^{*}$ INR: international normalization ratio.

${ }^{\dagger}$ GIB: gastrointestinal bleeding.

${ }^{\ddagger}$ NSAID: nonsteroidal anti-inflammatory drug.

TABle 6: Multivariate analysis of risk factors of gastrointestinal bleeding in patients taking warfarin.

\begin{tabular}{lccc}
\hline Variable & Relative risk & $\begin{array}{c}95 \% \\
\text { confidence } \\
\text { interval }\end{array}$ & $P$ value \\
\hline Age $>65$ yrs & 2.5 & $1.2-5.5$ & 0.02 \\
Mean INR ${ }^{*}>2.1$ & 2.1 & $1.0-4.2$ & 0.04 \\
History of $\mathrm{GIB}^{\dagger}$ & 5.1 & $1.9-13.5$ & 0.001 \\
Cirrhosis & 6.9 & $2.0-24.5$ & 0.003 \\
\hline
\end{tabular}

* INR: international normalization ratio.

${ }^{\dagger}$ GIB: gastrointestinal bleeding.

guided dosing with genotype-guided dosing of warfarin in patients initiating anticoagulant therapy, and genotypeguided dosing strategy did not result in a better outcome in both studies $[14,15]$. Therefore, close monitoring of INR testing is still the most important issue in patients initiating warfarin anticoagulation.

Several risk factors were found to be associated with GIB in this study. The incidence of GIB increased with the increment of the mean INR values, and a mean INR of 2.1 best discriminated the patients with and without GIB and patients with a mean INR of 3.0 or more carried the highest risk of GIB. This was not surprising because intensity of anticoagulation highly correlates with the risk of bleeding in warfarin anticoagulated patients in most studies $[4,5,16]$. Age $>65$ years old was found to be significantly associated with GIB. Slow metabolized rate of warfarin, an elevated risk of drug interactions because of polypharmacy, and chronic illness were proposed to increase the risk of bleeding in elderly patients [17]. Whether older age increases the risk of bleeding in patients treated with warfarin is controversial [5]. However, it is well known that persons older than 80 or 85 years of age do carry a significant risk of bleeding $[17,18]$. Therefore, warfarin should be cautiously used in elderly patients, especially in extremely old patients. Chronic liver disease had been shown to increase bleeding risks in hospitalized patients [19]. Although the mechanism is unclear, the relative deficiency in vitamin $\mathrm{K}$ dependent clotting factors causing bleeding tendency as seen in cirrhotic patients may play a role. We also found that a history of GIB increased the risk of GIB. Actually, concurrent use of proton pump inhibitors significantly reduces risk of GIB in patients treated with warfarin [20]. Therefore, acid suppressants may be considered in patients with a history of GIB during warfarin anticoagulation.

Most of the GIB events were controlled by vitamin K administration, blood transfusion, acid suppression, or endoscopic therapies. However, two fatal GIB events were noticed within the first month of warfarin anticoagulation. Both patients had a history of GIB and underlying liver cirrhosis. This suggested that patients with multiple risk factors of GIB might need more frequent testing of INR values, especially in the early stage of warfarin anticoagulation.

The pros and cons of restarting warfarin in the patients with GIB are rarely evaluated. It was noteworthy that warfarin was restarted in only $61.1 \%$ of our GIB patients and $27.3 \%$ of them experienced recurrent GIB while thromboembolic 
events were noticed in $16.7 \%$ of the patients who did not continue warfarin therapy. The chance of rebleeding after successful hemostasis depends on the cause of bleeding [4], and patients with independent risk factors for GIB should be thoroughly evaluated before restarting warfarin. Longterm acid suppressants may be considered in patients with peptic ulcer bleeding. Besides, a policy of lower-intensity anticoagulation may be beneficial in most of the cases. A warfarin dose adjusted to maintain an INR of 1.4 or more was found to be effective in the primary prevention of coronary heart disease [21]. Recent guidelines also recommended using lower-intensity anticoagulation in patients older than 75 years of age [22]. To prevent the thromboembolic event while minimizing the risk of GIB, an anticoagulation intensity at an INR of 2.0 or less could be an alternative anticoagulation strategy to traditional range, that is, 2.0 to 3.0.

Some limitations do exist in this study. First, it was a retrospective study and the GIB rate may be underestimated because some GIB patients might not present to this hospital. Second, the pharmacogenetics information such as polymorphisms in VKORC1 and CYP2C9 genes was not available in this study. Third, only $70 \%$ of our GIB patients underwent endoscopy, which was similar to a previous study [3]. Besides, the bleeding source was not identified in $12 \%$ of the patients undergoing endoscopic examination. Excessive anticoagulation intensity may contribute to the low diagnostic rate of endoscopy [23]. Fourth, some patients were missing the information of the status of Helicobacter pylori (H. pylori) infection. Patients with $H$. pylori infection are well known to be at a higher risk of GIB. Whether eradication of $H$. pylori can decrease the risk of GIB in warfarin anticoagulated patients needs to be studied in the future. Finally, although cirrhosis and a history of GIB were found to be independent risk factors for GIB, the possibility of overinterpretation could not be overlooked because the numbers of these patients were relatively small and the confidence intervals were wide during subgroup analysis.

\section{Conclusions}

Our study found that warfarin therapy carried a similar risk of GIB in Taiwanese patients as compared with most Western studies. Old age, intensity of anticoagulation, a history of GIB, and advanced liver disease were associated with GIB. To decrease the risk of GIB while maintaining the effect of anticoagulation, frequent testing of INR values and a strategy of low to moderate intensity of anticoagulation could be considered in patients with risk factors of GIB. In terms of restarting warfarin following hemostasis in patients with GIB, long-term acid suppressants could be used in addition to more close monitoring of INR values and low to moderate intensity of anticoagulation.

\section{Conflict of Interests}

The authors declare that the study was conducted in the absence of any commercial or financial relationships that could be construed as a potential conflict of interests.

\section{References}

[1] J. Hirsh, V. Fuster, J. Ansell, and J. L. Halperin, "American Heart Association/American College of Cardiology foundation guide to warfarin therapy,' Circulation, vol. 107, no. 12, pp. 1692-1711, 2003.

[2] L. G. Jacobs, "Warfarin pharmacology, clinical management, and evaluation of hemorrhagic risk for the elderly," Cardiology Clinics, vol. 26, no. 2, pp. 157-167, 2008.

[3] T. A. Rubin, M. Murdoch, and D. B. Nelson, "Acute GI bleeding in the setting of supratherapeutic international normalized ratio in patients taking warfarin: endoscopic diagnosis, clinical management, and outcomes," Gastrointestinal Endoscopy, vol. 58, no. 3, pp. 369-373, 2003.

[4] C. P. Choudari and K. R. Palmer, "Acute gastrointestinal haemorrhage in patients treated with anticoagulant drugs," Gut, vol. 36, no. 4, pp. 483-484, 1995.

[5] C. S. Landefeld and R. J. Beyth, "Anticoagulant-related bleeding: clinical epidemiology, prediction, and prevention," The American Journal of Medicine, vol. 95, no. 3, pp. 315-328, 1993.

[6] C. Becattini, M. C. Vedovati, and G. Agnelli, "Old and new oral anticoagulants for venous thromboembolism and atrial fibrillation: a review of the literature," Thrombosis Research, vol. 129, no. 3, pp. 392-400, 2012.

[7] I. L. Holster, V. E. Valkhoff, E. J. Kuipers, and E. T. T. L. Tjwa, "New oral anticoagulants increase risk for gastrointestinal bleeding: a systematic review and meta-analysis," Gastroenterology, vol. 145, no. 1, pp. 105-112, 2013.

[8] S. V. Shah and B. F. Gage, "Cost-effectiveness of dabigatran for stroke prophylaxis in atrial fibrillation," Circulation, vol. 123, no. 22, pp. 2562-2570, 2011.

[9] A. Blann, J. Hewitt, F. Siddiqui, and D. Bareford, "Racial background is a determinant of average warfarin dose required to maintain the INR between 2.0 and 3.0," British Journal of Haematology, vol. 107, no. 1, pp. 207-209, 1999.

[10] H. G. Xie, R. B. Kim, A. J. J. Wood, and C. M. Stein, "Molecular basis of ethnic differences in drug disposition and response," Annual Review of Pharmacology and Toxicology, vol. 41, pp. 815850, 2001.

[11] M. T. Lee, C. H. Chen, C. H. Chou et al., "Genetic determinants of warfarin dosing in the Han-Chinese population," Pharmacogenomics, vol. 10, no. 12, pp. 1905-1913, 2009.

[12] A. K. Daly, "Optimal dosing of warfarin and other coumarin anticoagulants: the role of genetic polymorphisms," Archives of Toxicology, vol. 87, no. 3, pp. 407-420, 2013.

[13] M. Wadelius, L. Y. Chen, J. D. Lindh et al., "The largest prospective warfarin-treated cohort supports genetic forecasting," Blood, vol. 113, no. 4, pp. 784-792, 2009.

[14] S. E. Kimmel, B. French, S. E. Kasner et al., "A pharmacogenetic versus a clinical algorithm for warfarin dosing," The New England Journal of Medicine, vol. 369, no. 24, pp. 2283-2293, 2013.

[15] M. Pirmohamed, G. Burnside, N. Eriksson et al., "A randomized trial of genotype-guided dosing of warfarin," The New England Journal of Medicine, vol. 369, no. 24, pp. 2294-2303, 2013.

[16] F. J. M. Van der Meer, F. R. Rosendaal, J. P. Vandenbroucke, and E. Briet, "Bleeding complications in oral anticoagulant therapy: an analysis of risk factors," Archives of Internal Medicine, vol. 153, no. 13, pp. 1557-1562, 1993.

[17] S. D. Fihn, C. M. Callahan, D. C. Martin, M. B. McDonell, J. G. Henikoff, and R. H. White, "The risk for and severity of bleeding 
complications in elderly patients treated with warfarin," Annals of Internal Medicine, vol. 124, no. 11, pp. 970-979, 1996.

[18] M. C. Fang, Y. Chang, E. M. Hylek et al., "Advanced age, anticoagulation intensity, and risk for intracranial hemorrhage among patients taking warfarin for atrial fibrillation," Annals of Internal Medicine, vol. 141, no. 10, pp. 745-752, 2004.

[19] C. S. Landefeld, E. F. Cook, M. Flatley, M. Weisberg, and L. Goldman, "Identification and preliminary validation of predictors of major bleeding in hospitalized patients starting anticoagulant therapy," The American Journal of Medicine, vol. 82, no. 4, pp. 703-713, 1987.

[20] K. J. Lin, S. Hernándezdíaz, and L. A. García Rodríguez, "Acid suppressants reduce risk of gastrointestinal bleeding in patients on antithrombotic or anti-inflammatory therapy," Gastroenterology, vol. 141, no. 1, pp. 71-79, 2011.

[21] P. K. MacCallum, P. J. Brennan, and T. W. Meade, "Minimum effective intensity of oral anticoagulant therapy in primary prevention of coronary heart disease," Archives of Internal Medicine, vol. 160, no. 16, pp. 2462-2468, 2000.

[22] L. S. Wann, A. B. Curtis, K. A. Ellenbogen et al., "Management of patients with atrial fibrillation (compilation of 2006 ACCF/AHA/ESC and 2011 ACCF/AHA/HRS recommendations): a report of the american college of cardiology/American Heart Association Task Force on practice guidelines," Circulation, vol. 127, no. 18, pp. 1916-1926, 2013.

[23] C. M. Wilcox and C. D. Truss, "Gastrointestinal bleeding in patients receiving long-term anticoagulant therapy," The American Journal of Medicine, vol. 84, no. 4, pp. 683-690, 1988. 


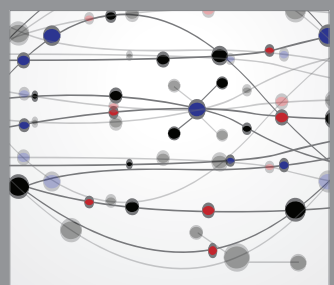

The Scientific World Journal
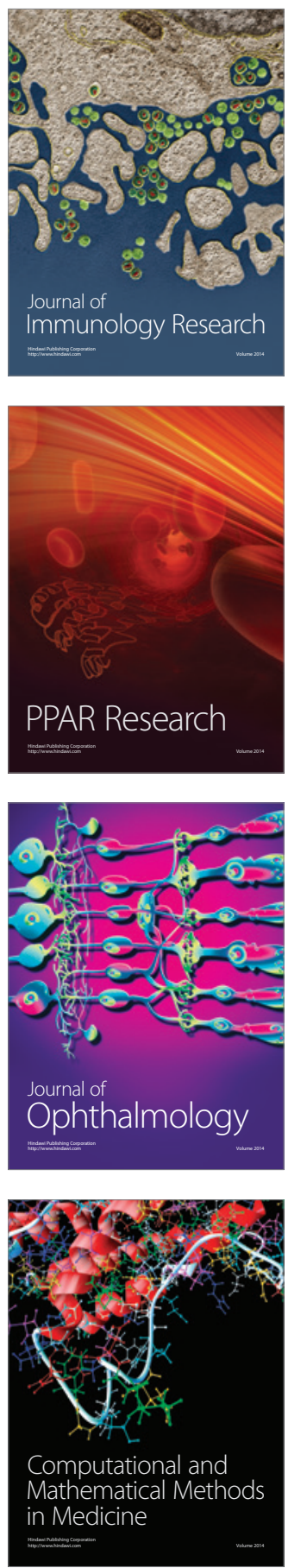

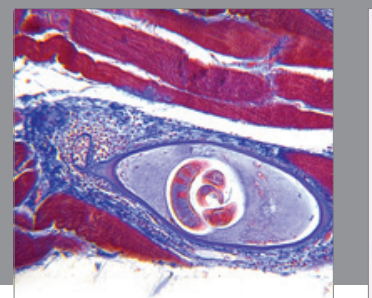

Gastroenterology

Research and Practice
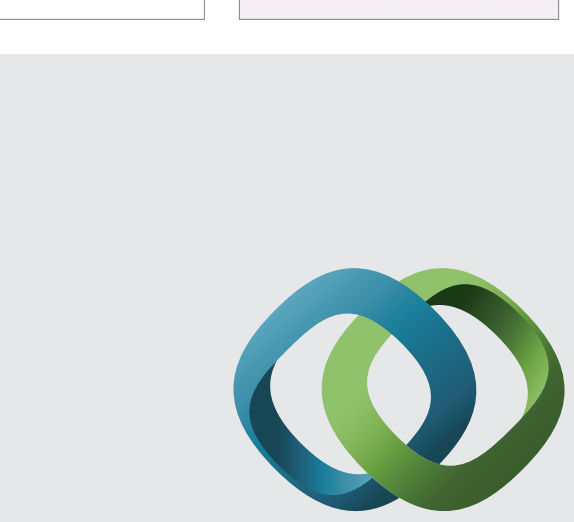

\section{Hindawi}

Submit your manuscripts at

http://www.hindawi.com
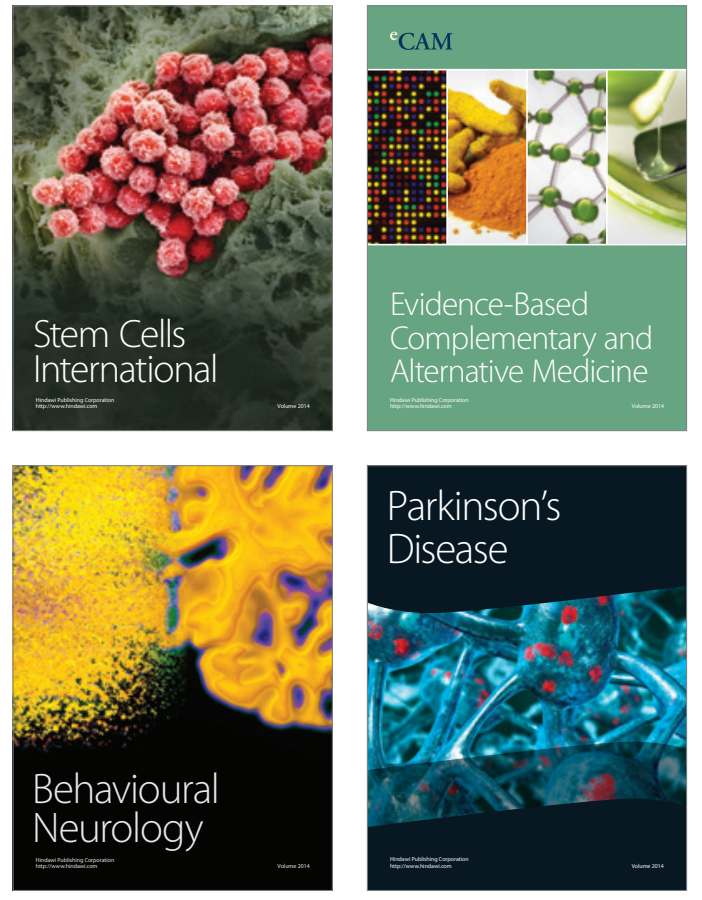
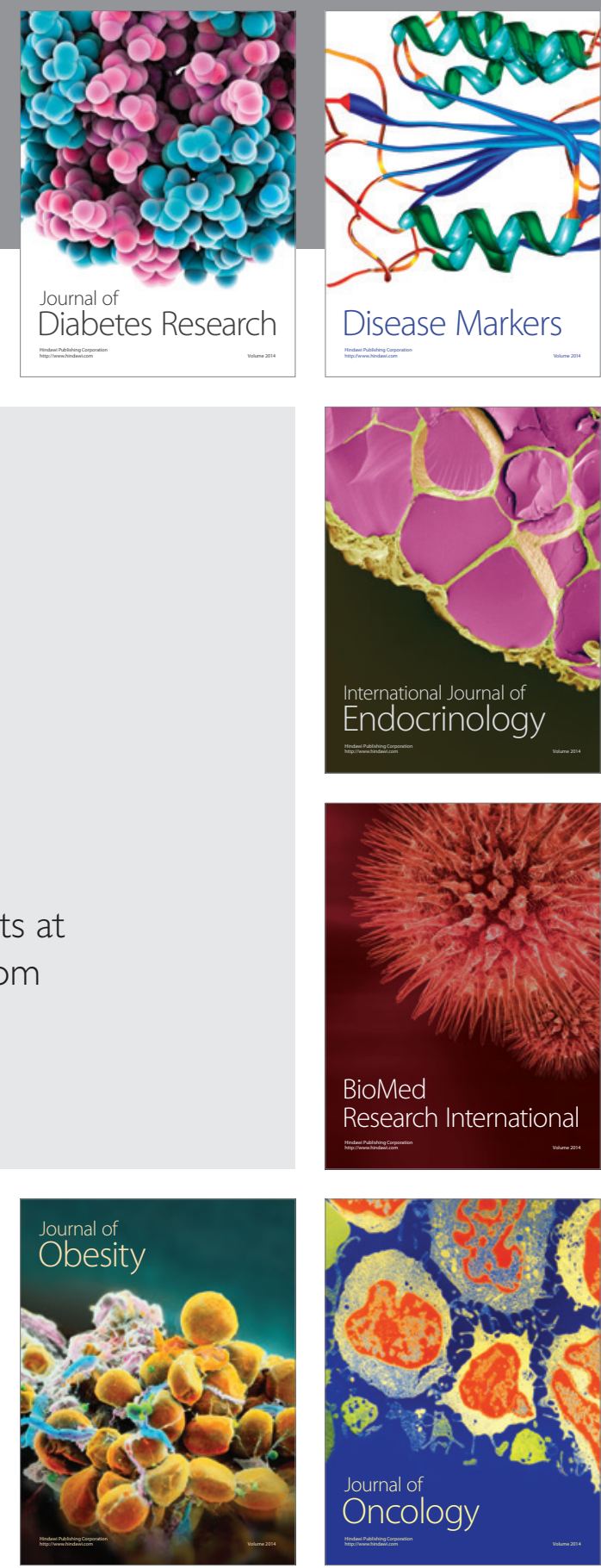

Disease Markers
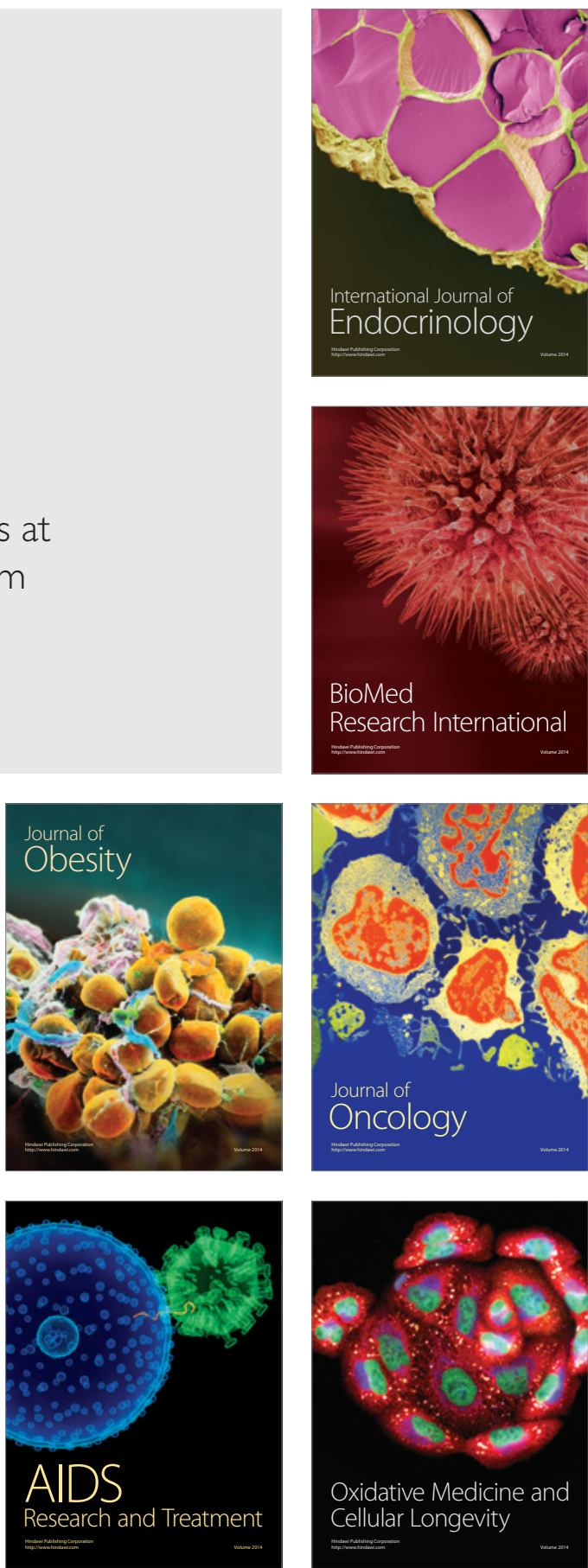